\title{
A High-Granularity Timing Detector for the ATLAS Phase-II upgrade
}

\author{
Chiara Rizzi ${ }^{a, b, *}$, on behalf of the ATLAS Collaboration \\ ${ }^{a}$ CERN, Geneva, Switzerland \\ ${ }^{b}$ Departement de Physique Nucleaire et Corpusculaire, Université de Genève, Geneva, Switzerland \\ E-mail: chiara.rizzi@cern.ch
}

\begin{abstract}
The High-Luminosity (HL) phase of the Large Hadron Collider (LHC) at CERN will offer a great opportunity to consolidate and expand the LHC physics program, but it will also present several experimental challenges. One of the major ones is the increase in the number of pile-up interactions in each bunch crossing. The High Granularity Timing Detector (HGTD) is a Phase-II ATLAS upgrade that will use timing information to reduce the impact of pile-up, covering the pseudorapidity region between 2.4 and 4.0 and providing a per-track time resolution of $30 \mathrm{ps}$ and $50 \mathrm{ps}$ respectively at the beginning and at the end of the HL-LHC operation.
\end{abstract}

40th International Conference on High Energy physics - ICHEP2020

July 28 - August 6, 2020

Prague, Czech Republic (virtual meeting)

\footnotetext{
${ }^{*}$ Speaker
} 


\section{Introduction}

During the high-luminosity (HL) phase, the Large Hadron Collider (LHC) at CERN [1] is expected to deliver an integrated luminosity of $4000 \mathrm{fb}^{-1}$. In order to achieve this goal, the instantaneous luminosity will be raised to $7.5 \times 10^{34} \mathrm{~cm}^{-2} \mathrm{~s}^{-1}$, an increase of more than a factor three with respect to the Run 2 conditions, that implies an increase in pile-up, with an average of simultaneous $p p$ interactions in the same bunch crossing $(<\mu>)$ up to 200. In the period of time without collisions preceding the beginning of the HL phase (Long Shutdown 2, LS2), the ATLAS detector [2] will be upgraded to be able to face the new challenges of this increased luminosity.

The High-Granularity Timing Detector (HGTD) is a Phase-II upgrade that will improve the ATLAS functionality in the forward region in the high pile-up environment of the HL-LHC. The main goal of HGTD is to limit the negative impact of the increased pile-up on the ATLAS physics performance, and improve the ATLAS capabilities in terms of physics object reconstruction in the forward region. To reject pile-up, from the purely spatial point of view, the main handle is the impact parameter along the beam axis $\left(z_{0}\right)$. The uncertainty on $z_{0}$, without the HGTD contribution, degrades noticeably in the high-pseudorapidity region, especially for particles with low transverse momentum $\left(p_{\mathrm{T}}\right)$. HGTD protects the performance in the forward region: with a per-track time resolution of $30 \mathrm{ps}$ and $50 \mathrm{ps}$ respectively at the beginning and at the end of the detector lifetime, it provides precise time information that allows adding one extra dimension, the time, to resolve the hard scattering vertex and therefore to mitigate the pile-up impact.

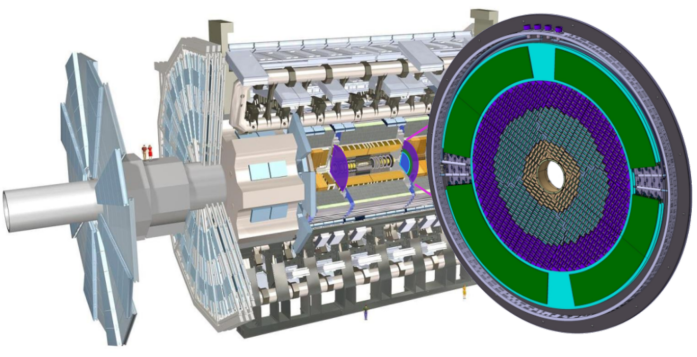

(a)

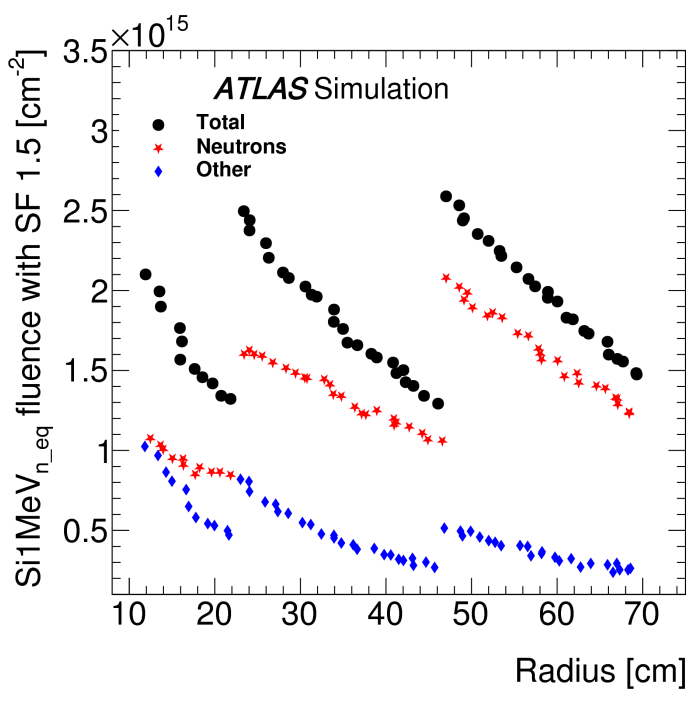

(b)

Figure 1: (a) Location of HGTD inside the ATLAS detector. (b) Expected fluence at each radius, taking into account a safety factor of 1.5 and the plan for the ring replacements. Figures from Ref. [3]. 


\section{HGTD Layout}

The basic unit of the HGTD are the modules; the bare module consists of a large sensor, of $30 \times 15$ pixels, bump-bonded to two ASICs. The bare module, together with the flexible printed circuit board (flex cables) that connects it to the peripheral electronics, is the HGTD hybrid module. Modules are hosted on support units, to aid in the assembly process.

The HGTD will be located in the region between the barrel and the end-cap calorimeters (approximately 3.5 meters from the interaction point), and its radius will extend from 11 to $100 \mathrm{~cm}$. The location of the two HGTD vessels inside ATLAS is shown in Figure 1(a). Each vessel contains two double-sided layers and two moderators. Each layer has modules on both sides, and the modules are partially overlapping. The active area of each layer is divided into three rings $(120 \mathrm{~mm}<\mathrm{r}<$ $230 \mathrm{~mm}, 230 \mathrm{~mm}<\mathrm{r}<470 \mathrm{~mm}$, and $470 \mathrm{~mm}<\mathrm{r}<640 \mathrm{~mm}$, covering a pseudorapidity region $2.4<|\eta|<4.0$ ) and the amount of overlap between the front and backside modules decreases with the increase of the radius. This ring structure has the advantage of allowing an easy replacement of the sensors at a small radius, which will suffer higher radiation damage. The inner ring will be replaced each $1000 \mathrm{fb}^{-1}$, the middle ring each $2000 \mathrm{fb}^{-1}$, the outer ring will never be replaced. These replacements allow keeping the total dose that each sensor receives lower than $2.5 \times 10^{15}$ $\mathrm{n}_{\mathrm{eq}} \mathrm{cm}^{-2}$ (including a safety factor of 1.5 to account for uncertainties in the simulation), as shown in Figure 1(b). This allows achieving both high-enough collected charge in each sensor, and lowenough ionizing dose on the ASICS. With this layout, the expected number of hits from each track is $\geq 2$ for all the active area of the detector. In order to reach the goal of a per-track time resolution (30 ps at the beginning of HL-LHC, $50 \mathrm{ps}$ at the end) this requires a per-hit time resolution of approximately $45 \mathrm{ps}$ and $70 \mathrm{ps}$ for un-irradiated and irradiated sensors respectively.

\section{Sensors}

The HGTD sensors are based on the Low Gain Avalance Detector (LGAD) technology, originally developed by the Centro Nacional de Microelectronica (CNM) Barcelona [4]. These are n-on-p silicon detectors, with a highly-doped layer that causes internal charge amplification (gain). LGAD sensors from different vendors (CNM, HPK, FBK, and recently from NDL) and with different features are considered in the HGTD Technical Design Report [3]. The baseline pixel has been chosen to be $1.3 \mathrm{~mm} \times 1.3 \mathrm{~mm}$, with an active thickness of $50 \mu \mathrm{m}$.

The sensor performance has been tested before and after irradiation with protons and neutrons, both with laboratory studies and with beam tests. Figure 2 shows examples of performance results for sensors from different vendors (CNM, HPK, and FBK) as a function of the bias voltage at the maximum fluence of $2.5 \times 10^{15} \mathrm{n}_{\mathrm{eq}} \mathrm{cm}^{-2}$. In the Figure, the red line shows the target detector performance: a collected charge greater than $4 \mathrm{fC}$ (to have a high signal efficiency and a low jitter form the ASICs) and a per-hit time resolution of $70 \mathrm{ps.} \mathrm{While} \mathrm{the} \mathrm{R \& D} \mathrm{on} \mathrm{the} \mathrm{sensors} \mathrm{is} \mathrm{still}$ ongoing, it is possible to observe that the current sensors already provide the required performance also after irradiation. 


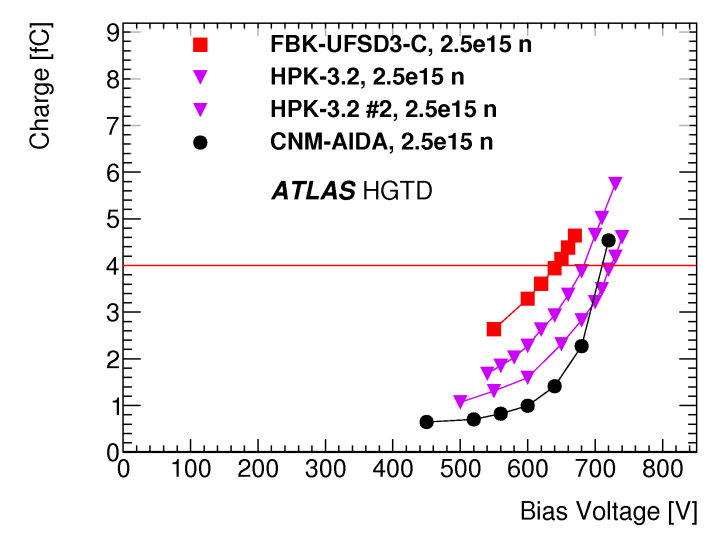

(a)

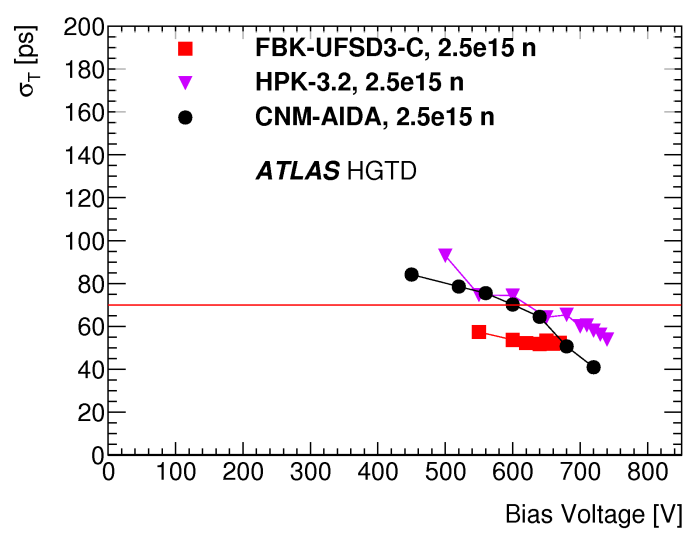

(b)

Figure 2: Collected charge (a) and time resolution (b) as a function of the bias voltage at the maximum fluence of $2.5 \times 10^{15} \mathrm{n}_{\mathrm{eq}} \mathrm{cm}^{-2}$. Figures from Ref. [3].

\section{ASICs}

The LGAD sensors are read out by dedicated ASICs (ALTIROC), whose design is driven by the main requirements of radiation resistance and low jitter. The ALTIROC will provide two times: the time of arrival (TOA) and the time over threshold (TOT), which is used as a proxy to the signal amplitude and to correct the TOA for time walk effects.

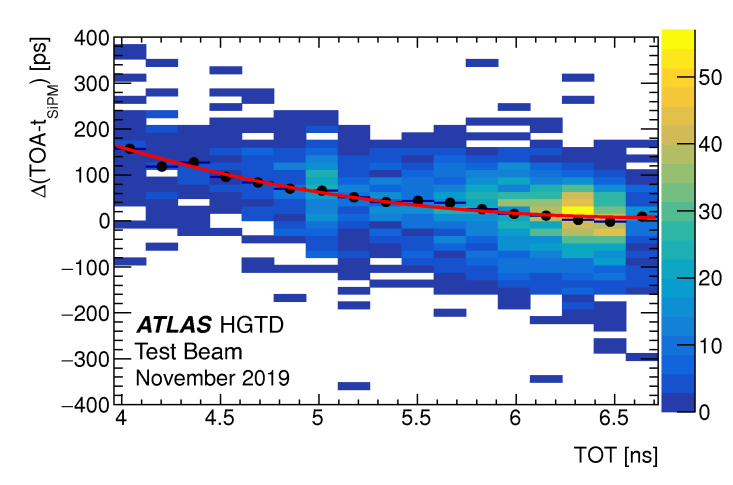

(a)

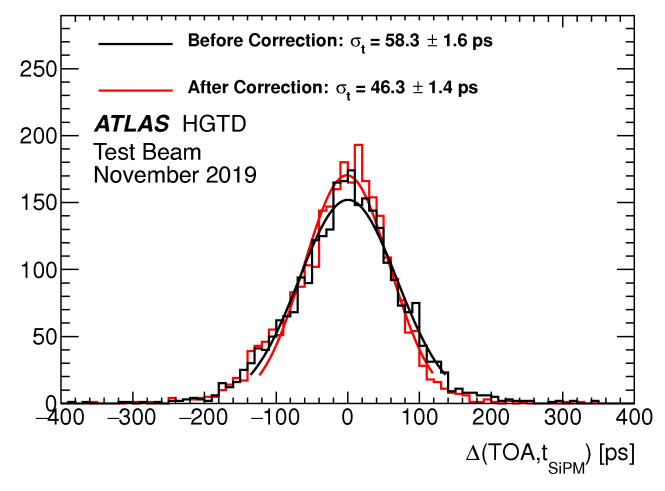

(b)

Figure 3: (a) Distribution of the TOA as a function of the TOT. (b) Distribution of the difference in time between the LGAD+ALTORIC1 system and the reference silicon photomultiplier Figures from Ref. [6].

The ALTIROC performance has been studied both in the laboratory and with beam tests. Figure 3 shows the results of the test of ALTIROC1 (a 25-channel ALTIROC prototype) at DESY with $5 \mathrm{GeV}$ electrons [5]. Figure 3(b) shows the distribution of TOA as a function of the TOT, used to derive the time walk correction. Figure 3(a) shows that the difference in time between the LGAD+ALTORIC1 system and the reference silicon photomultiplier has a width of 48 and $46 \mathrm{ps}$ respectively before and after the correction for time walk. Subtracting a Landau contribution of 25 
ps leaves with a time resolution of $39 \mathrm{ps}$ from jitter, clock and TDC. This performance is affected by a noise source coming from the DAQ board, and is expected to be reduced to less than $30 \mathrm{ps}$ with the recently developed filtering interface.

\section{Impact on Physics Performance}

The most obvious handle to reject pile-up is to identify the time of the hard scatter vertex $\left(t_{0}\right)$, and require that the other tracks that are used to build physics objects have an associated time consistent with $t_{0}$. In this respect, HGTD can help to identify pile-up jets. Other ways in which pile-up tracks can decrease the performance is for example when a pile-up track causes an electron to fail the isolation requirement (and HGTD would therefore improve the electron isolation efficiency) or when it decreases the flavor-tagging performance.

Another important aspect of the HGTD improvements to the ATLAS performance is related to the luminosity measurement. HGTD is designed to have high granularity, which in turns leads to low occupancy, and therefore good linearity between average number of hits and the instantaneous luminosity, as shown in Figure 4.

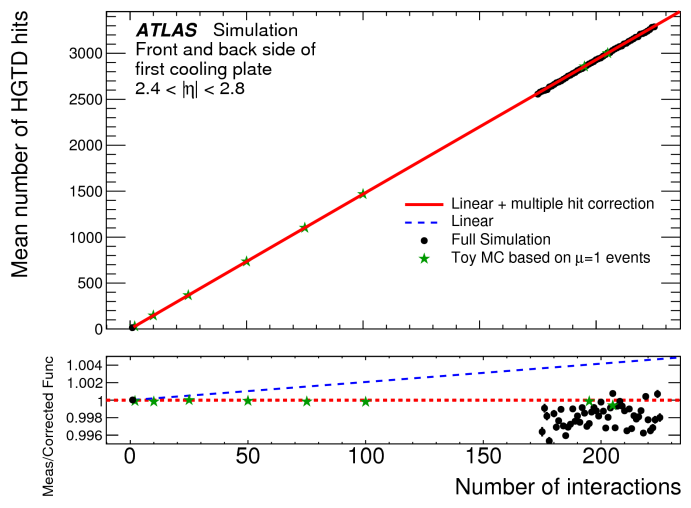

(a)

Figure 4: (a) Linearity of the average number of hits as a function of the number of interactions. Figure from Ref. [3].

\section{Conclusion}

The High Granularity Timing Detector is a Phase-II upgrade for the ATLAS Experiment, designed to mitigate the impact of increased pile-up in the HL-LHC, where it is expected to have up to 200 simultaneous $p p$ interactions per bunch crossing. The detector is optimized to achieve a per-track time resolution of $30 \mathrm{ps}$ at the beginning of the HL-LHC, and $50 \mathrm{ps}$ at the end. The performance of the detector components and the impact of the ATLAS physics program have been studied and are documented in the Technical Design Report [3]. 


\section{References}

[1] G. Apollinari et al., High-Luminosity Large Hadron Collider (HL-LHC): Technical Design Report V. 0.1, CERN Yellow Reports: Monographs, CERN, 2017, URL: http://cds.cern.ch/record/2284929.

[2] ATLAS Collaboration, The ATLAS Experiment at the CERN Large Hadron Collider. JINST 3 (2008) S08003.

[3] ATLAS Collaboration, Technical Design Report: A High-Granularity Timing Detector for the ATLAS Phase-II Upgrade, CERN-LHCC-2020-007, URL: https://cds.cern.ch/record/2719855.

[4] G. Pellegrini et al., Technology developments and first measurements of Low Gain Avalanche Detectors (LGAD) for high energy physics applications, Nucl. Instrum. Meth. A765 (2014) 12.

[5] R. Diener et al., The DESY II test beam facility, Nuclear Instruments and Methods in Physics Research Section A: Accelerators, Spectrometers, Detectors and Associated Equipment 922 (2019) 265, ISSN: 0168-9002, URL: http://www.sciencedirect.com/science/article/pii/S0168900218317868.

[6] C. Agapopoulou et al., Performance of a Front End prototype ASIC for picosecond precision time measurements with LGAD sensors, (2020), arXiv: 2002.06089 [physics.ins-det]. 